\title{
Analysis of Combined Convection in an Open Cavity under Constant Heat Flux Boundary Conditions and Magnetic Field Using Finite Element Method
}

\author{
A. K. Azad ${ }^{1 *}$, M. J. H. Munshi' ${ }^{2}$, M. M. Rahman ${ }^{3,4}$, and M. K. Chowdhury ${ }^{3}$ \\ ${ }^{1}$ Department of Business Administration, International Islamic University Chittagong, Bangladesh \\ ${ }^{2}$ Department of Mathematics, Hamdard University Bangladesh, Gozaria, Munshiganj, Bangladesh \\ ${ }^{3}$ Department of Mathematics, Bangladesh University of Engineering and Technology, Dhaka-1000, \\ Bangladesh \\ ${ }^{4}$ Department of Electrical Engineering, Faculty of Engineering, University of Malaya, 50603, Kuala \\ Lumpur, Malaysia
}

Received 8 April 2013, accepted in final revised form 5 April 2014

\begin{abstract}
This study investigated the effects of the aspect ratio of the cavity for average fluid temperature at exit port, average Nusselt number, maximum temperature of the fluid in the domain, drag coefficient, isotherms and streamlines on behalf of different Hartmann numbers and Rayleigh numbers. Solution of governing equations of momentum and energy has been made by finite element technique. Above mentioned parameters such as an aspect ratio which is cavity height to cavity length change from $A r=0.5$ to 2 for different Rayleigh numbers and Hartmann numbers which change from $R a=10^{3}$ to $10^{5}$ and $H a=0$ to 50 respectively. Prandtl number $P r=7$ and Reynolds number $R e=100$ is fixed in this simulation. It is found that variation of the aspect ratio makes an important effect for higher values of Rayleigh numbers. Heat transfer enhances with increasing of aspect ratio. Increasing of Hartmann number decreases the heat transfer inside the cavity.
\end{abstract}

Keywords: Temperature boundary conditions; Open cavity; Aspect ratio; Finite element methods.

(c) 2014 JSR Publications. ISSN: 2070-0237 (Print); 2070-0245 (Online). All rights reserved.

doi: http://dx.doi.org/10.3329/jsr.v6i2.14505 J. Sci. Res. 6 (2), 243-256 (2014)

\section{Introduction}

Heat and fluid flow from blocked or open cavities are important applications for cooling of electronic equipments, solar energy applications, nuclear reactors and building HVAC systems. In these systems, the heat transfer regime can be forced or natural convection depends on the flow velocity and magnetic field. They can be control parameters for heat and fluid flow. There is a big challenge on cooling of electronic equipments to enhance efficiency of these devices as indicated by Nasrin [1], Hsu and How [2], Kieno et al. [3],

\footnotetext{
* Corresponding author: azadmacu@gmail.com
} 
House et al. [4], Dong and Li [5]. A numerical investigation of mixed convection in a ventilated square cavity with a heat conducting horizontal solid circular cylinder has been performed by Rahman et al. [6]. In rectangular cavities at different phase ratios, Gau et al . [7] conducted mixed convection with moving isothermal side walls and constant flux as a heat source on the bottom wall. Nasrin and Alim [8] numerically studied transient falling behavior of a sand grain in the presence of Dufour and Soret effects. They considered flow, temperature and concentration fields in terms of streamlines, isotherms and isoconcentration, respectively for various dimensionless times until the steady state was observed. They got the results from their numerical analysis that the heat transfer rate is more effective than the rate of mass transfer. Heat transfer results for mixed convection from a bottom heated open cavity subjected to an external flow are reported by Leong et al. [9] for different Reynolds number, Grashof numbers and aspect ratios. They found that Reynolds number and Grashof number control the flow pattern and occurrence of recirculating cells while the aspect ratio has a significant influence on the orientation of these cells. Rahman et al. [10] investigated the effects of magnetic field on mixed convection heat and mass transfer in an open cavity for fixed aspect ratio and constant temperature boundary conditions. They observed that heat transfer decreases with increasing of Hartmann number. Mixed convection flow and heat transfer enhancement in an inclined lid-driven triangular enclosure filled with nanofluids is studied numerically by Billah et al. [11]. The flow and heat transport structures are presented in terms of streamlines and isotherms, respectively in their study. Oztop and Dagtekin [12] examined a vertical two-sided lid-driven differentially heated square cavity with steady state twodimensional mixed convection problem numerically. They found that the fluid flow and the heat transfer are affected by both Richardson number and direction of moving walls in the cavity. The effects of viscous dissipation on unsteady combined convective heat transfer to water near its density maximum in a rectangular cavity with isothermal wall was investigated by Hossain and Gorla [13]. The problem of the Prandtl number effect on forced convection heat transfer in a wavy open cavity filled with water- $\mathrm{CuO}$ nanofluid has been studied numerically by Parvin et al. [14]. Flow and temperature field in terms of streamlines and isotherms have been displayed in their study. Combined heat and mass transfer from a horizontal channel with an open cavity heated from below is numerically examined by Brown and Lai [15]. They used scale analysis to correlate the parameters. Buscaglia and Dari [16] studied a problem of open channel under volumetric heat source in a cavity. They observed two distinct regimes are identified, one dominated by inertia in which significant hot-fluid trapping occurs, and another, a stratified regime, in which buoyancy effects are dominant. A computational study is performed by Parvin and Nasrin [17] to investigate the mixed convection in an octagonal channel with a heat-generating horizontal circular body. Results are obtained for wide ranges of Reynolds number, Richardson number and Prandtl number. Mixed convection in a partially divided rectangular enclosure over a wide range of Reynolds and Grashof numbers was studied by Calmidi and Mahajan [18]. They got the average Nusselt number and the dimensionless surface temperature depended on the position and height of the dividing wall. Open cavity 
flow is also found in electronic block arrays. In this context, Davalath and Bayazitoglu [19] made a work by considering the each block under constant heat flux boundary conditions. Then, some of the studies are focused on to enhance heat transfer in blocked channels. A computational study is performed by Nasrin [20] to analyze the aspect ratio effect of a vertical lid driven chamber in presence of magnetic field. Rahman et al. [21] worked on the conjugated effect of joule heating and magneto-hydrodynamic on doublediffusive mixed convection in a horizontal channel with an open cavity. joule heating parameter has a little effect on buoyancy-induced vortex in the streamlines and concentration contours. Manca et al. [22] presented a numerical simulation of laminar mixed convection in a channel with an open cavity and heated wall bounded by a horizontal insulated plate. Further a study of magnetohydrodynamic mixed convection around a heat conducting horizontal circular cylinder placed at the center of a rectangular cavity along with joule heating has been carried out by Rahman et al. [23]. On the other hand, it has a striking effect on isotherms and density contours. Type of boundary conditions plays important role in energetical systems, especially on short conduits and cavities. In this context, the local heat transfer coefficients for isothermal and uniform heat flux boundary conditions for a planar wall jet have been determined by Abdul Nour et al. [24]. They made several measurements for different parameters. They observed that the local heat transfer coefficient is insensitive to the thermal boundary condition at sufficiently large downstream locations where the outer turbulent layer has diffused into the inner region of the wall jet. These two fundamental temperature boundary conditions were compared for circular free impinging jet and a solid surface with non-uniform wall temperature or wall heat flux by Wang et al. [25-26].

This paper is an extension of the work of Manca et al. [22] incorporating the influence of magnetic field. The method of finite element analysis is applied to solve the nonlinear partial differential equations. The main aim of the present work is to make a presentation on the effects of the aspect ratio, magnetic field on constant heat flux boundary conditions in an open enclosure with the presence of buoyancy forces. Results will be presented via streamlines, isotherms, average Nusselt number and drag forces. Based on above literature survey and author's knowledge, number of studies on the comparison of effects of thermal boundary conditions for open channel is extremely limited.

\section{Model and Governing Equations}

Physical model is presented in Fig. 1 with dimensions and boundary conditions. It is an open cavity heated from the bottom with constant heat flux boundary conditions. Remaining walls are adiabatic and all walls are impermeable. Flow inlets to the channel via uniform temperature and velocity. The uniform magnetic field $B_{0}$ is also applied opposite to the fluid flow direction and parallel to the $\mathrm{x}$-axis. It is assumed that the induced magnetic field produced by the motion of an electrically conducting fluid is negligible compared to the applied magnetic field. $\mathrm{L} / \mathrm{H}$ value defines the aspect ratio (Ar) which changes between 0.5 and 2 . 


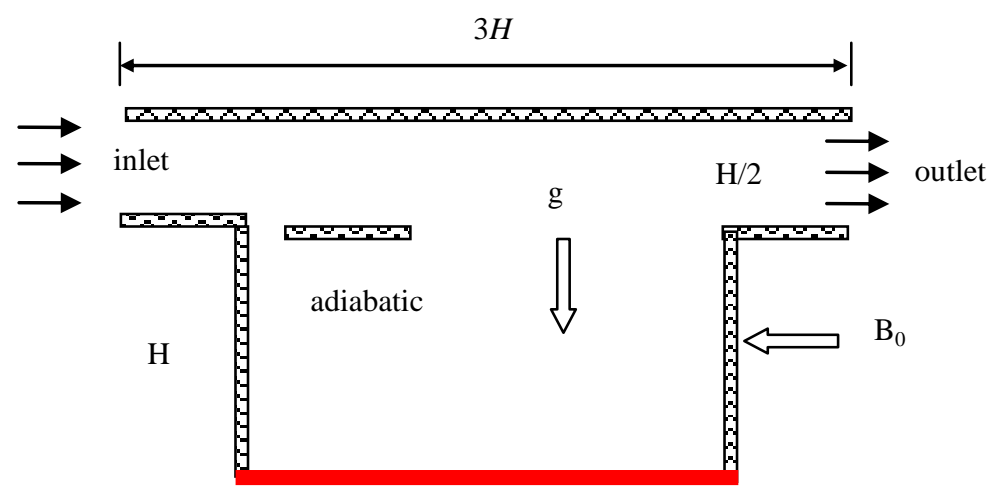

$q, L$

Fig. 1. Physical model of the problem with boundary conditions.

A two-dimensional, steady, laminar, incompressible, mixed convection flow is considered within the cavity as well as channel, and the fluid properties are assumed to be constant. The radiation effects are taken as negligible. The dimensionless equations describing the flow under Boussinesq approximation are as follows [21, 23]:

$$
\begin{aligned}
& \frac{\partial U}{\partial X}+\frac{\partial V}{\partial Y}=0 \\
& U \frac{\partial U}{\partial X}+V \frac{\partial U}{\partial Y}=-\frac{\partial P}{\partial X}+\frac{1}{R e}\left(\frac{\partial^{2} U}{\partial X^{2}}+\frac{\partial^{2} U}{\partial Y^{2}}\right) \\
& U \frac{\partial V}{\partial X}+V \frac{\partial V}{\partial Y}=-\frac{\partial P}{\partial Y}+\frac{1}{R e}\left(\frac{\partial^{2} V}{\partial X^{2}}+\frac{\partial^{2} V}{\partial Y^{2}}\right)+\frac{R a}{R e^{2} P r} \theta-\frac{H a^{2}}{R e} V \\
& U \frac{\partial \theta}{\partial X}+V \frac{\partial \theta}{\partial Y}=\frac{1}{\operatorname{RePr}}\left(\frac{\partial^{2} \theta}{\partial X^{2}}+\frac{\partial^{2} \theta}{\partial Y^{2}}\right)
\end{aligned}
$$

where $R e=\frac{u_{i} H}{v}, \operatorname{Pr}=\frac{v}{\alpha}$ and $R a=\frac{g \beta q H^{4}}{k v \alpha}$ are Reynolds number, Prandtl number and Rayleigh number respectively and $H a$ is Hartmann number which is defined as $H a^{2}=\frac{\sigma B_{0}^{2} H^{2}}{\mu}$.

The above equations were non-dimensionalized by using the following dimensionless quantities $X=\frac{x}{H}, Y=\frac{y}{H}, U=\frac{u}{u_{i}}, V=\frac{v}{u_{i}}, P=\frac{p+\rho g y H^{2}}{\rho u_{i}{ }^{2}}$, and $\theta=\frac{T-T_{i}}{q H / k}$

where $X$ and $Y$ are the coordinates varying along horizontal and vertical directions respectively, $U$ and $V$ are the velocity components in the $X$ and $Y$ directions respectively, $\theta$ is the dimensionless temperature and $\mathrm{P}$ is the dimensionless pressure

The boundary conditions for the present problem are specified as follows: 
at the inlet: $U=1, V=0, \theta=0$

at the outlet: $\frac{\partial U}{\partial X}=0, V=0, \frac{\partial \theta}{\partial X}=0$

at all solid boundaries other than bottom wall: $U=0, V=0, \frac{\partial \theta}{\partial N}=0$

at the bottom wall: $U=V=0, \frac{\partial \theta}{\partial Y}=-1$

where $N$ is the non-dimensional distances either $X$ or $Y$ direction acting normal to the surface.

The average Nusselt number at the heated surface is calculated as $N u_{a v}=-\int_{0}^{1} \frac{1}{\theta} d X$, and the average drag coefficient is, $D=-\int_{0}^{1} \frac{\partial U}{\partial Y} d X$.

\section{Solution}

The Galerkin weighted residual method of finite element formulation is used as a numerical procedure in this work. The finite element method begins by the partition of the continuum area of interest into a number of simply shaped regions called elements. These elements may be different shapes and sizes. Within each element, the dependent variables are approximated using interpolation functions. In the present study the computational domain was discretized with a smooth mesh of triangular elements. The coupled governing equations (2)-(4) are transformed into sets of algebraic equations using finite element method to reduce the continuum domain into discrete triangular domains. The system of algebraic equations is solved by applying Newton-Raphson iteration technique. The solution process is iterated until the subsequent convergence condition is satisfied:

$\left|\Gamma^{m+1}-\Gamma^{m}\right| \leq 10^{-6}$ where $\mathrm{n}$ is number of iteration and $\Gamma$ is the general dependent variable.

A grid independence study was executed to make sure the correctness of the numerical results for a square cavity $(A r=1)$ at the representative value of $R a=10^{5}, H a=10$. Table 1 shows the convergence of average Nusselt number, $N u_{\mathrm{av}}$ with grid refinement. It is observed that the magnitude of the average Nusselt number for 5120 elements shows a very little difference with the results obtained for the other higher elements. The element 5120 is chosen for further calculation of the case $A r=1$. This grid resolution is therefore used for all consequent calculations for $A r<1$ and $A r>1$.

Table 1 . Grid sensitivity check at $R a=10^{5}, H a=10$ and $A R=1$.

\begin{tabular}{ccccc}
\hline Elements & 2906 & 5120 & 8106 & 9636 \\
$N u_{\text {av }}$ & 2.638961 & 2.646872 & 2.646974 & 2.64633 \\
\hline
\end{tabular}

Due to validate our numerical model we have compared obtained results with the most closely related numerical solution of Manca et al. [22] for mixed convection problem in a channel with an open cavity. The comparison is conducted applying dimensionless 
parameters $\operatorname{Pr}=0.71, R e=100, R i=0.1, w / H=0.5$ and $L / H=2$ which is shown in Table 2. Present result is an excellent agreement with those of Manca et al. [22] in which maximum difference within $0.5 \%$. These validations make a well assurance in this numerical code.

Table 2. Comparison of results for validation at $\operatorname{Pr}=0.71, R e=100, R i=0.1$, $w / H=0.5, L / H=2$.

\begin{tabular}{ccc}
\hline Opposing flow & Present & Manca et al. [22] \\
\hline$N u_{\mathrm{av}}$ & 1.7657 & 1.7748 \\
$\theta_{\max }$ & 0.629 & 0.627 \\
\hline
\end{tabular}

\section{Results and Discussion}

A numerical analysis has been conducted using finite element method in a heated open cavity under constant heat flux boundary conditions with different Rayleigh numbers and aspect ratios. The Effects of magnetic field also analyzed for different Hartmann numbers.

In Fig. 2 (a) to (c) present streamlines (on the top) and isotherms (on the bottom) for different Hartmann numbers, which change from $H a=0$ to 50 at $A r=2$ and $R a=10^{5}$ under constant heat flux boundary conditions. General observations shows in figures that a circulation occurs inside the cavity but its strength change with Hartmann number. For $H a=0$, the fluid flow directly above the cavity and stream function value is formed as 0.21 . In this case, the cavity is heated from the bottom under constant heat flux and isotherms cluster near the right bottom corner due to impingement of fluid to right vertical wall. Isotherms inside the cavity resemble to the heated cavity problem with natural convection. With increasing of $\mathrm{Ha}$ number, the center of the circulation cell moves to left side and stream function is obtained as -0.04. Also Sinusoidal shaped temperature distribution is observed from the isotherms, and almost parallel temperature distribution is observed near the bottom wall as in Fig. 2 (b). This cell disappeared and only a small circulation cell is formed at the left bottom corner which rotates in the clockwise direction. The corresponding isotherms are almost cavity-shaped, which is evocative of the supremacy of conduction heat transfer in the cavity but almost parallel to the horizontal surface in the channel as in Fig. 2 (c). It means that heat transfer becomes weaker inside the cavity with increasing of Hartmann number.

Figs. 3 (a) - (c) show the streamlines (on the top) and isotherms (on the bottom) for different values of Hartmann number $H a=0,25$ and 50 at $A r=1.5$ and $R a=10^{5}$. As seen from Fig. 3 (a) a clockwise circulation occurs inside the cavity, the fluid flow directly above the cavity and stream function value is formed as -0.208 . Isotherms are almost clustered near the right bottom corner. In Fig. 3(b and c) circulation inside the cavity is suppressed and moves to left side accordingly, also stream function value is obtained as 0.04 and -0.01 respectively. On the other hand isotherms are almost curvature in the cavity. Fig. 4 ( $a, b$ and c) shows the streamlines and isotherms for different values of Hartmann number $H a=0,25$ and 50 at $A r=1$ and $R a=10^{5}$. As seen from the Fig. 4 (a) an amount of fluid near the heating wall of the cavity is activated so as to create a 
buoyancy-induced clockwise rotating cell and isotherms show the usual convective twist inside the cavity for the lowest value of $\mathrm{Ha}=0$.

(a)

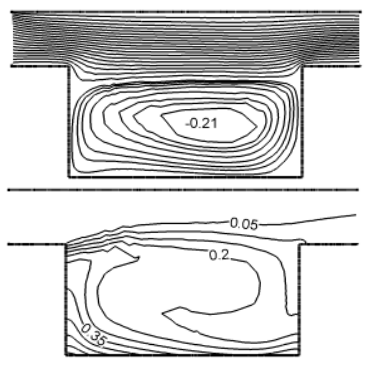

(b)

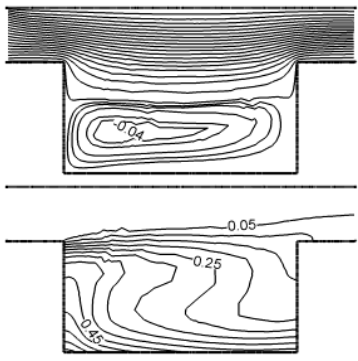

(c)

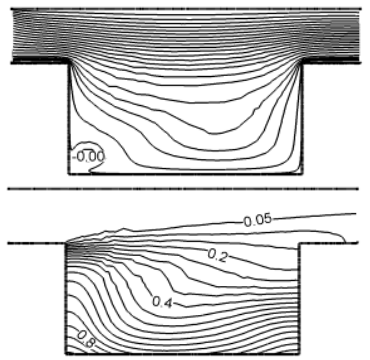

Fig. 2. Streamlines (on the top) and Isotherms (on the bottom) for $A r=2, R a$ $=10^{5}$ and (a) $H a=0$, (b) $H a=25$, (c) $H a$ $=50$. (a)

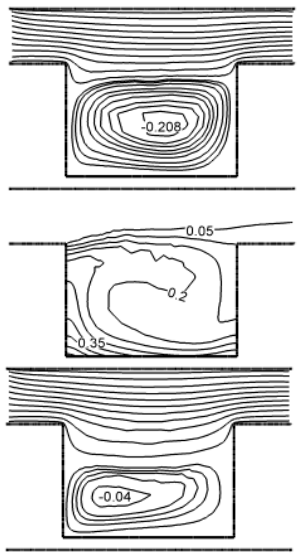

(b)

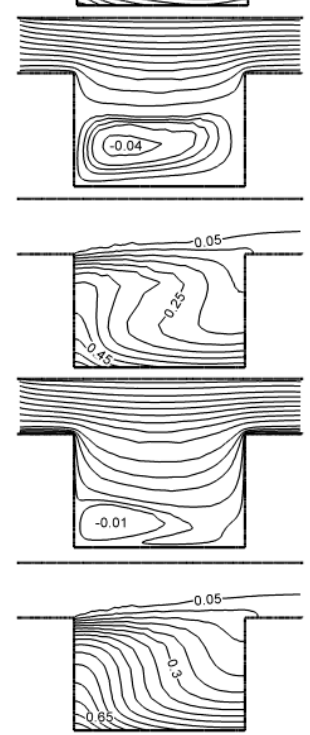

Fig. 3. Streamlines (on the top) and Isotherms (on the bottom) for $A r=1.5$, $R a=10^{5}$ and (a) $H a=0$, (b) $H a=25$, (c) $H a=50$.

The distortion of isothermal lines appears due to the high convective current inside the cavity. As the Hartmann number increases, the strength of the rotating cell is reduced and pushed to the left bottom corner of the cavity(Fig. 4 (b)) and then through flow in the channel gains its strength and occupies the whole of the cavity as well as the channel indicating the establishment of conduction mode of heat transfer( Fig. 4 (c)). Distortions of isothermal lines start to disappear with increasing Hartmann number (Fig. 4 (b)). As Hartmann number increases, isothermal lines inside the cavity as well as the channel approaches more and more towards the conduction-like distribution pattern of isothermal lines. For large Hartmann number $\mathrm{Ha}=50$ (Fig. 4(c)) the convection is almost suppressed, 
and the isotherms are almost parallel to the horizontal wall, indicating that a quasiconduction regime is reached.

(a)

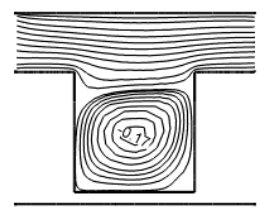

(b)
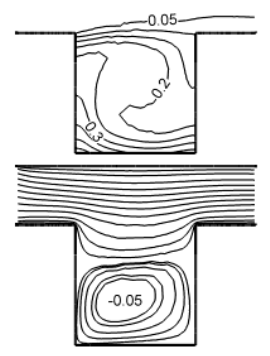

(c)

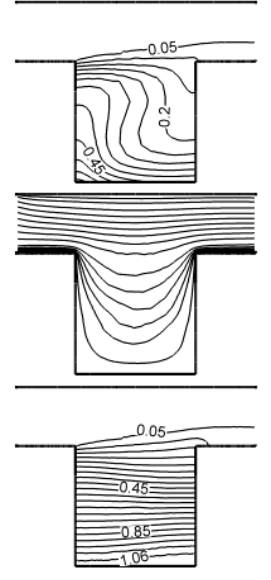

Fig. 4. Streamlines (on the top) and Isotherms (on the bottom) for $A r=1, R a=$ $10^{5}$ and (a) $H a=0$, (b) $H a=25$, (c) $H a=$ 50 . (a)

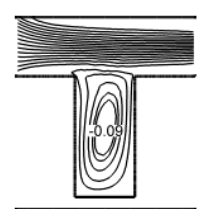

(b)

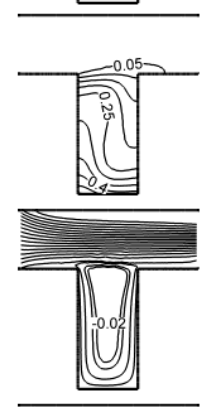

(c)

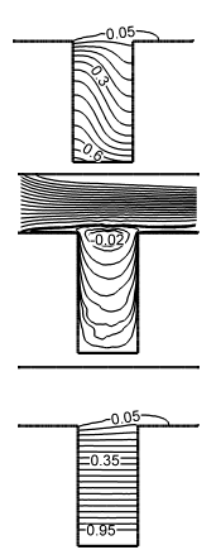

Fig. 5. Streamlines (on the top) and Isotherms (on the bottom) for $A r=0.5$, $\mathrm{Ra}=10^{5}$ and (a) $H a=0$, (b) $H a=25$, (c) $H a=50$.

In case of $A r=0.5$ and $R a=10^{5}$ which are presented in Fig. 5(a, b and c). For $H a=0$, the flow behaves as lid-driven cavity, but it can be controlled by magnetic field. As given in Fig. 5 (c), temperature is the distribution as parallel to flow and bottom wall. It means that conduction mode heat transfer becomes dominant.

Fig. 6(a) presents average Nusselt number for different Hartmann numbers at $R a=$ $10^{5}$. As seen from the figure, average Nusselt number increases with increasing of aspect ratio. Dissimilar trend is observed for Hartmann numbers, i.e. average Nusselt number 
decreases with the increase of Hartmann number. Fig. 6(b) presents the maximum fluid temperature versus cavity aspect ratio for different $\mathrm{Ha}$ and $\mathrm{Ra}=10^{5}$. From the figure we can say that the maximum fluid temperature decreases for $A r=$ to 0.5 to 1 but increases for $A r=1$ to 2 for different Hartmann numbers except $H a=50$. For $H a=50$, the maximum fluid temperature increases slowly for $A r=0.5$ to 1 , decreases for $A r=1$ to 1.5 and then increases for 1.5 to 2 .

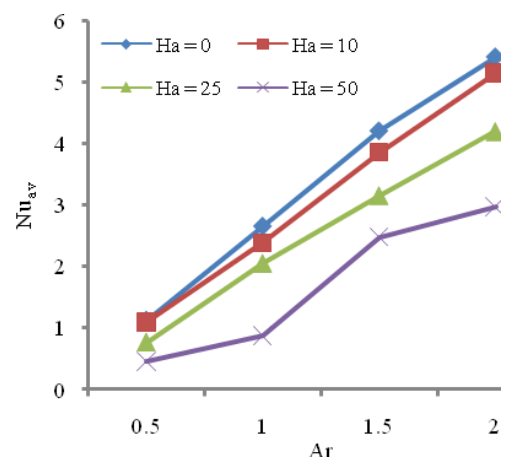

Fig. 6. (a) Average Nusselt number versus cavity aspect ratio for different $\mathrm{Ha}$ and $\mathrm{Ra}$ $=10^{5}$.

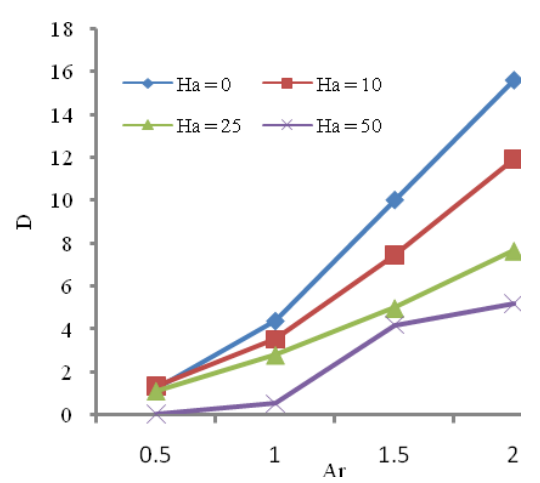

Fig. 7. (a) Average drag coefficient versus cavity aspect ratio for different $\mathrm{Ha}$ and $\mathrm{Ra}=$ $10^{5}$.

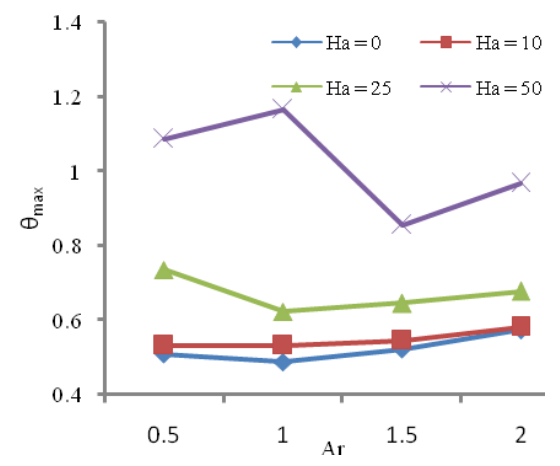

Fig. 6. (b) Maximum fluid temperature versus cavity aspect ratio for different $\mathrm{Ha}$ and $R a=10^{5}$.

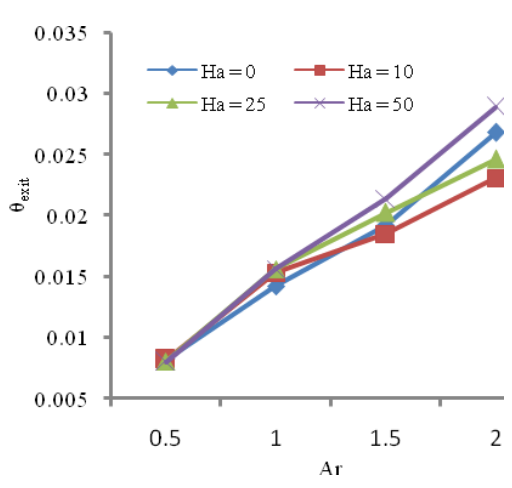

Fig. 7. (b) Fluid temperature at the exit port versus cavity aspect ratio for different $\mathrm{Ha}$ and $R a=10^{5}$.

Fig. 7(a) illustrates the variation of average drag coefficient with aspect ratio at different Hartmann numbers for $R a=10^{5}$. Average drag coefficient show similar behavior with Nusselt number. As given in the figure, values are almost zero for $A r=0.5$ due to motionless fluid but it increases with the increase of aspect ratio. Higher drag coefficient values are obtained than the lower value of Hartmann number. Fig. 7(b) illustrates the 
variation of fluid temperature at exit port versus cavity aspect ratio for different Hartmann number and $R a=10^{5}$. Fluid temperatures at exit port are almost same for lower values of Ar. On the other hand, the values become different for the higher values of $A r$.

Fig. 8 illustrates the streamlines (on top) and isotherms (bottom) for different Rayleigh numbers and $H a=10$ and $A r=2$. As seen from the figures, there is no circulating cell inside the cavity for lower values of Rayleigh number $\left(R a=10^{3}\right)$ as in Fig. 8 (a). Flowing fluid inside the enclosure hits fluid to right vertical wall and flow strength decreases as seen from Fig. 8(b). Similar distribution is formed for $R a=10^{5}$. In this case, buoyancy induced flow becomes effective as seen from Fig. 8 (c).

(a)

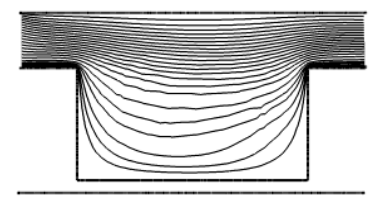

(b)
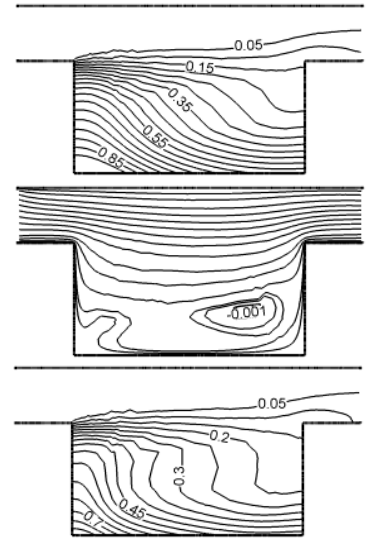

(c)

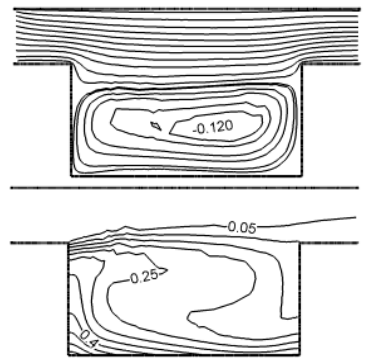

Fig. 8. Streamlines (top) and isotherms (bottom) for $A r=2, H a=10$ and (a) $R a=10^{3}$, (b) $\mathrm{Ra}=10^{4}$, (c) $R a=10^{5}$. (a)

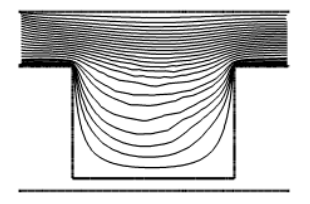

(b)

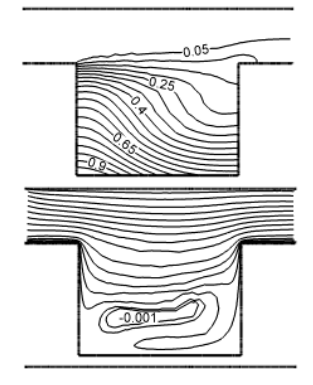

(c)

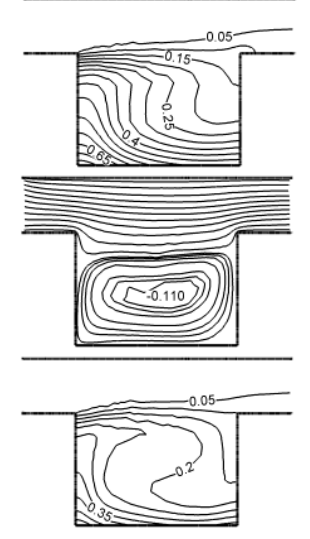

Fig. 9. Streamlines (top) and isotherms (bottom) for $A r=1.5, H a=10$ and (a) $\mathrm{Ra}=$ $10^{3}$, (b) $R a=10^{4}$, (c) $R a=10^{5}$.

Fig. 9 presents streamlines and isotherm results for different Rayleigh numbers at $A r=$ 1.5 and $H a=10$. Figures show that streamlines and isotherms illustrate almost the same distribution as Fig. 8. Flow strength decreases with the increase of Rayleigh numbers. At 
low Rayleigh number, flowing fluid compresses the fluid inside the cavity. Convection heat transfer becomes stronger at high Rayleigh numbers and flowing fluid along the channel moves directly above the cavity.

Fig. 10 illustrate the results for $A r=1$ and $H a=10$ for the same parameters as Fig. 9. As seen from results at $A r=1$, flow strength decreases for all values of Rayleigh numbers. Also, shape of circulation cell turns from elliptic to circle inside the open cavity. It is also seen that the isotherms are almost parallel to the bottom wall for the lower values of $R a$ and becomes convection dominated with increasing $R a$.

The effects of Rayleigh number on streamlines and isotherms at $\mathrm{Ha}=10$ are presented in Fig. 11 for $A r=0.5$. The figures also can be compared with Fig. 5 for different Hartmann numbers and $R a=10^{5}$. Thus, Fig. 11 gives that the effects of Rayleigh number at $A r=0.5$ show similar distribution as do other cases. It is seen that convection heat transfer becomes higher for $H a=10$. The inside of the cavity is more heated with the increase of Rayleigh number and it affects the flowing fluid along the channel.

(a)

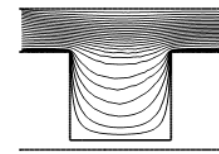

(b)
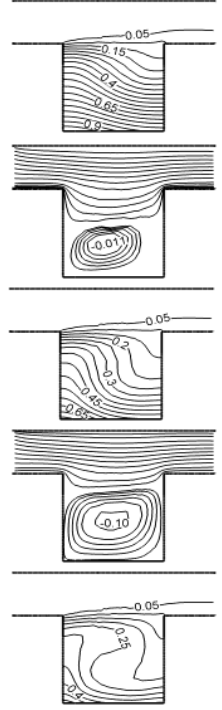

Fig. 10. Streamlines (top) and isotherms (bottom) for $A r=1, H a=10$ and (a) $R a$ $=10^{3}$, (b) $R a=10^{4}$, (c) $R a=10^{5}$. (a)

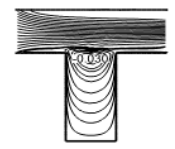

(b)
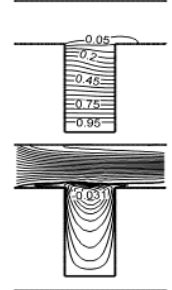

(c)

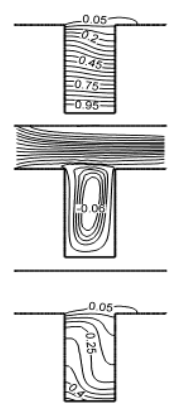

Fig. 11. Streamlines (top) and isotherms (bottom) for $\mathrm{Ar}=0.5, \mathrm{Ha}=10$ and (a) $\mathrm{Ra}=$ $10^{3}$, (b) $R a=10^{4}$, (c) $R a=10^{5}$.

Fig. 12 (a) illustrates the variation of average Nusselt number with aspect ratio at different Rayleigh numbers for $H a=10$. The figure compares the average Nusselt numbers with different Rayleigh number. As seen from the figure, a linear increasing 


\section{Analysis of Combined Convection}

occurs on average Nusselt number with aspect ratio. As expected results, heat transfer corresponds with the increase of Rayleigh numbers. Fig. 12(b) shows the variation of maximum fluid temperature versus $A r$ for different $R a$ and $H a=10$. Maximum fluid temperature $\left(\theta_{\max }\right)$ goes down very slowly with increasing $\mathrm{Ar}$ for lower values of $\operatorname{Ra}\left(10^{3}\right.$ and $10^{4}$ ) but at $A r=0.5$ the maximum fluid temperature values are same. Also the temperature increases very slowly with $A r$ at the highest value of $R a$.

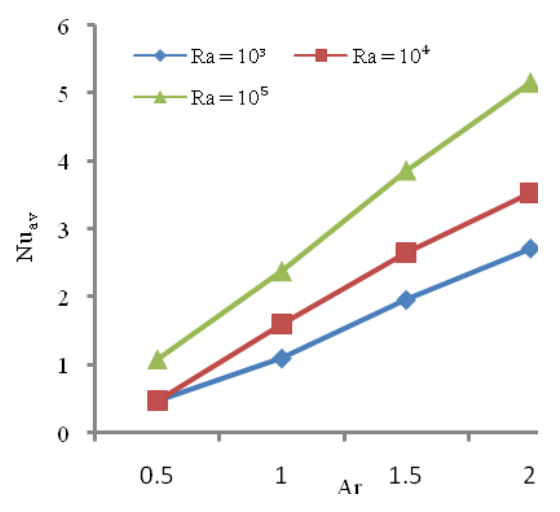

Fig. 12. (a) Average Nusselt number and versus cavity aspect ratio for different $\mathrm{Ra}$ and $H a=10$.

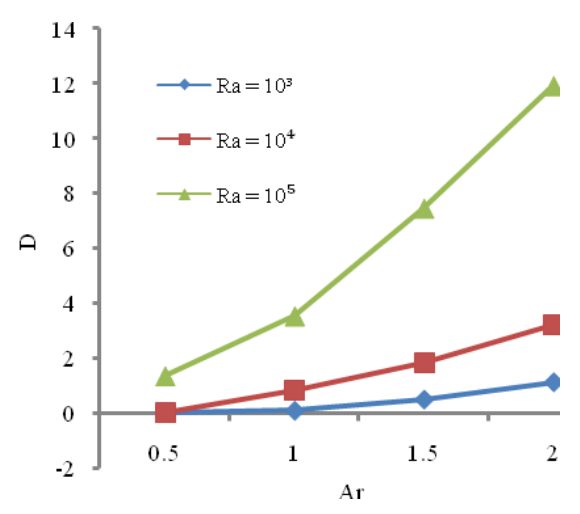

Fig. 13. (a) Average drag coefficient versus cavity aspect ratio for different for different $\mathrm{Ra}$ and $H a=10$

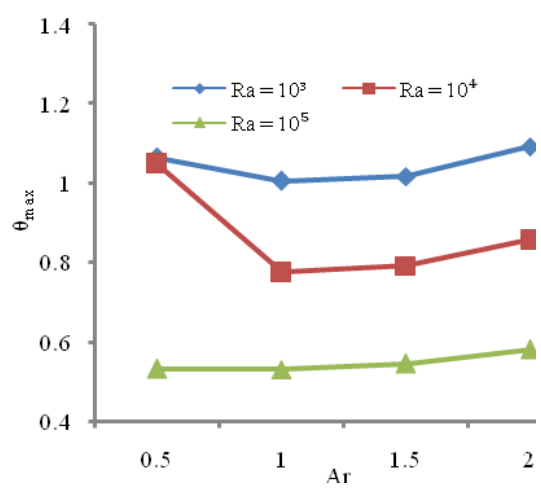

Fig. 12. (b) Maximum fluid temperature versus cavity aspect ratio for different $\mathrm{Ra}$ and $H a=10$.

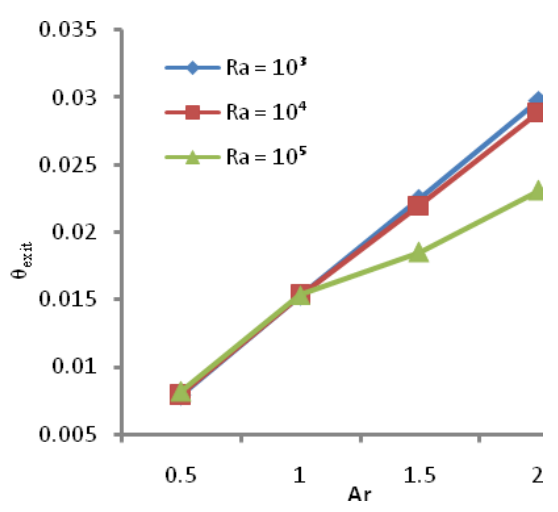

Fig. 13. (b) Fluid temperature at the exit port versus cavity aspect ratio for different for different $\mathrm{Ra}$ and $\mathrm{Ha}=10$

Fig 13 (a) gives the average drag coefficient with aspect ratio at different Rayleigh numbers for $\mathrm{Ha}=10$. Average drag coefficient shows similar behavior with Nusselt number. As given in the figure, the values are almost zero for $\mathrm{Ar}=0.5$ due to motionless 
fluid but it increases with the increase of aspect ratio. Higher drag force values are obtained for higher values of Ra. Fluid temperature at the outlet of the channel is presented for same parameters with Figs. 12 and 13(a) in Fig. 13(b). But average temperature at the exit increases with the increase of cavity aspect ratio. The figure also shows that effects of Rayleigh number become insignificant at outlet average temperature due to low convection.

\section{Conclusions}

This study presents the results of solving of governing equations for a heated cavity from the bottom wall under magnetic field for different Rayleigh numbers and aspect ratios. Some important findings can be listed as follows.

(a) Average Nusselt number, drag coefficient and average temperature at the exit increase with the increase of aspect ratio $(A r)$.

(b) At $A r=0.5$, flow becomes almost motionless at low values of Rayleigh numbers.

(c) Single cell was formed inside the enclosure almost for all cases except $\mathrm{Ha}=50$ due to domination of conduction mode of heat transfer.

(d) Single cell was found inside the enclosure for the higher values of $R a$. Also conduction dominated heat transfer was found for the lower values of $R a$.

(e) Hartmann number decreases the flow strength and convection. Thus, the heat transfer regime becomes conduction for higher values of Hartmann numbers even at high Rayleigh numbers.

(f) The problem has relevance and significance in engineering and science.

\section{Nomenclature}

\begin{tabular}{|llll|}
\hline$A r$ & cavity aspect ratio & $u, v$ & dimensional velocity components $\left(\mathrm{ms}^{-1}\right)$ \\
$B_{0}$ & strength of the magnetic field, (Tesla) & $U, V$ & dimensionless velocity components \\
$D$ & average drag coefficient & $x, y$ & dimensional coordinates $(\mathrm{m})$ \\
$g$ & gravitational acceleration $\left(\mathrm{ms}^{-2}\right)$ & $X, Y$ & dimensionless coordinates \\
$H$ & height of the cavity $(\mathrm{m})$ & Greek symbols & \\
$\mathrm{Ha}$ & Hartmann number & $\alpha$ & thermal diffusivity \\
$k$ & fluid conductivity $\left(\mathrm{Wm}^{-1} \mathrm{k}^{-1}\right)$ & $\beta$ & thermal expansion coefficient \\
$L$ & length of the cavity $(\mathrm{m})$ & $\mu$ & dynamic viscosity $\left(\mathrm{kg}^{-1} \mathrm{~s}^{-1}\right)$ \\
$N$ & any direction & $v$ & kinematic viscosity \\
$N u$ & Nusselt number & $\theta$ & non-dimensional temperature \\
$p$ & dimensional pressure $\left(\mathrm{kgm}^{-1} \mathrm{~s}^{-2}\right)$ & $\rho$ & density $\left(\mathrm{kgm} \mathrm{m}^{-3}\right)$ \\
$P$ & non-dimensional pressure & $\sigma$ & electrical conductivity $\left(\mathrm{Sm}^{-1}\right)$ \\
$P r$ & Prandtl number & $\Gamma$ & general dependent variable \\
$q$ & uniform heat flux per unit area $\left(\mathrm{Wm}^{-2}\right)$ & Subscripts & \\
$R a$ & Rayleigh number & $i$ & inlet \\
$R e$ & Reynolds number & $h$ & hot \\
$T$ & Temperature $(\mathrm{K})$ & $a v$ & average \\
& & $\max$ & maximum \\
\hline
\end{tabular}




\section{References}

1. R. Nasrin, J. Sci. Res. 4 (1), 39 (2012). http://dx.doi.org/10.3329/jsr.v4i1.8014

2. T. H. Hsu and S. P. How, Acta Mechanica 133, 87 (1999). http://dx.doi.org/10.1007/BF01179012

3. P. F. Kieno, A. Ouédraogo, O. M. Zongo, J. D. Bathiébo and B. Zeghmati. J. Sci. Res. 5 (1), 105 (2013). http://dx.doi.org/10.3329/jsr.v5i1.10709

4. J. M. House, C. Beckerman and T. F. Smith, Numer. Heat Transfer (Part A) 18, 213 (1990). http://dx.doi.org/10.1080/10407789008944791

5. S. F. Dong and Y.T. Li, Int. J. of Heat and Mass Transfer 47, 2233 (2004). http://dx.doi.org/10.1016/j.ijheatmasstransfer.2003.11.018

6. M. M. Rahman, M. M. Billah and M. A. Alim, J. Sci. Res. 3 (2), 271 (2011). http://dx.doi.org/10.3329/jsr.v3i2.4344

7. G. Guo and M. A. R. Sharif, Int. J. Therm. Sci. 43 (5), 465 (2004). http://dx.doi.org/10.1016/j.ijthermalsci.2003.08.008

8. R. Nasrin and M. A. Alim, J. Sci. Res. 5 (1), 91 (2013).http://dx.doi.org/10.3329/jsr.v5i1.10003

9. J. C. Leong, N.M. Brown and F.C. Lai, Int. Comm. Heat Mass Transfer 32, 592 (2005). http://dx.doi.org/10.1016/j.icheatmasstransfer.2004.10.018

10. M. M. Rahman, S. Parvin, R. Saidur, and N.A. Rahim, Int. Comm. Heat Mass Transfer, 38, 193 (2011). http://dx.doi.org/10.1016/j.icheatmasstransfer.2010.12.005

11. M. M. Billah, M. M. Rahman, M. Shahabuddin and A. K. Azad, J. Sci. Res. 3 (2), 525 (2011).

12. H. F. Oztop and I. Dagtekin, Int. J. of Heat and Mass Transfer 47, 1761 (2004). http://dx.doi.org/10.1016/j.ijheatmasstransfer.2003.10.016

13. M. A. Hossain and R. S. R. Gorla, Int. J. of Numer. Methods for Heat and Fluid Flow 16 (1), 5 (2006). http://dx.doi.org/10.1108/09615530610636928

14. S. Parvin, R. Nasrin, M. A. Alim and N. F. Hossain, J. Sci. Res. 5 (1), 67 (2013). http://dx.doi.org/10.3329/jsr.v5i1.9641

15. N. M. Brown and F. C. Lai, Int. Comm. Heat Mass Transfer 32, 1008 (2005). http://dx.doi.org/10.1016/j.icheatmasstransfer.2004.10.029

16. G.C. Buscaglia and E.A. Dari, Numerical Heat Transfer (Part A) 43, 541 (2003). http://dx.doi.org/10.1080/10407780307317

17. S. Pervin and R. Nasrin, J. Sci. Res. 4 (2), 337 (2012). http://dx.doi.org/10.3329/jsr.v4i2.8142

18. V. V. Calmidi and R. L. Mahajan, Int. J. of Heat and Fluid Flow 19, 358 (1998). http://dx.doi.org/10.1016/S0142-727X(98)00002-2

19. J. Davalath and Y. Bayazitoğlu, J. Heat Transfer 109 (2), 328 (1987). http://dx.doi.org/10.1115/1.3248083

20. R. Nasrin, J. Sci. Res. 3 (3), 501 (2011). http://dx.doi.org/10.3329/jsr.v3i3.7433

21. M. M. Rahman, R. Saidur and N. A. Rahim, Int. J. Heat Mass Transfer 54, 3213 (2011) http://dx.doi.org/10.1016/j.ijheatmasstransfer.2011.04.010.

22. O. Manca, S. Nardini, K. Khanafer, and K. Vafi, Num. Heat Transfer, Part A 43, 282 (2003).

23. M. M. Rahman, M. A. Alim and M. K. Chowdhury, J. Sci. Res. 1 (3), 461 (2009). DOI: 10.3329 /jsr.v1i3.2597

24. R. S. Abdul Nour, K. Willenborg, J. J. McGrath, J. F. Foss, B. S. and A. Nour, Exp. Thermal Fluid Science 22, 131(2000). http://dx.doi.org/10.1016/S0894-1777(00)00018-2

25. X. S. Wang, Z. Dagan and L. M. Jiji, Int. J. Heat Mass Transfer 32, 1360 (1989). http://dx.doi.org/10.1016/0017-9310(89)90034-3

26. X. S. Wang, Z. Dagan and L. M. Jiji, Int. J. Heat Mass Transfer 32, 1371 (1989). http://dx.doi.org/10.1016/0017-9310(89)90035-5 